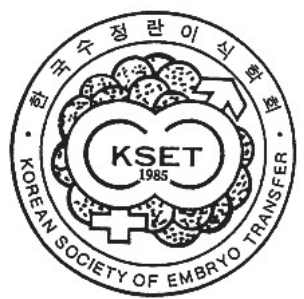

\title{
Ameliorative Effect of Chitosan Complex on Miniature Pig Sperm Cryopreservation
}

\author{
Hye-Min Hong, Ga-Young Sim, So-Mi Park, Eun-Joo Lee and Dae-Young Kim ${ }^{\dagger}$
}

Department of Life Science, College of Bio-nano technology, Gachon University, Seongnam 13120, Republic of Korea.

\section{Abstract}

Cryopreservation is mainly used for preservation of boar sperm. However, this method stresses the sperm by reactive oxygen species (ROS), and the conception rate and the litter size are not more efficient than the liquid preservation of spermatozoa. Therefore, we use chitosan which is a natural product derived antioxidant compound. We used GnHA (chitosan+hyaluronic acid) and GnHG (chitosan hydrogel) as chitosan complexes to cryopreserve boar sperm for improve sperm metabolism and function. Sperm parameter (sperm motility, progressive motility, path velocity, straight-line velocity, curvilinear velocity) is measured by computer-assisted sperm analysis (CASA) using frozen sperm with GnHA or GnHG $(0,0.25,0.5,1 \mathrm{mg} / \mathrm{mL})$, respectively. Also, lipid peroxidation analysis using malondialdehyde (MDA) is performed to confirm the antioxidative effect of chitosan in frozen spermatozoa. CASA analysis showed GnHA and GnHG are effective against cryopreserved boar sperm. And antioxidant effect is measured by lipid peroxidation analysis. GnHA and GnHG, which is chitosan complex are effective for boar sperm cryopreservation by antioxidant effect.

Received : 24 October 2018

Revised : 14 November 2018

Accepted : 18 December 2018
Key Words : Boar sperm, Chitosan, Cryopreservation, CASA, Lipid peroxidation

\footnotetext{
* This work was supported by the National Research Foundation of Korea (NRF) and Center for Women In Science, Engineering and Technology (WISET) grant funded by the Ministry of Science and ICT (MSIT) under the team research program for female engineering students.

+ Correspondence: Dae-Young Kim (ORCID: 0000-0003-1608-8441) 


\section{INTRODUCTION}

Despite the advance of modern medicine, the number of patients who need to receive an organ transplant has been steadily increasing, but the supply of organs is absolutely insufficient (Yun, 2016). Pigs are advantageous in that they can breed easily, their gestation period is short, and they bring forth large baby pigs. In addition, pigs are regarded to be a source of heterogeneous organs the most suitable for humans because most of the major organs of pigs are physiologically similar to human organs and are similar in size as well (Prather et al., 2003).

Since the conservation of bio-species can produce important properties of countries in relation to the Convention on Biological Diversity, the United Nations Convention on Biological Diversity (UNCBD) came into effect for the conservation of biodiversity. Therefore, the cryopreservation of pig sperms has been extensively studied in that it can store the sperms of good breeds of pigs for long periods of time and can be used for human xenogeneic organ transplantation.

Sperm freezing is the most efficient method of preserving the sperms of mammals, and in particular, the cryopreservation of pig sperms for artificial insemination has been carried out more widely compared to other domestic animals because artificial insemination through freezing enables mass production (Safranski et al., 2011; Yeste, 2016)

However, since pig sperms have a lower cholesterol / phospholipid ratio than other mammals such as humans, rams, stallions, and bulls, the cell membrane fluidity is low leading to difficulties in keeping the sperms in good conditions at low temperature (Schäfer et al., 2017). In addition, sperm storage at low temperatures can increase the production and stimulation of free radicals and high levels of free radicals have many adverse effects on cells, including DNA damage, lipid peroxidation and protein denaturation and are known to be a major cause of male infertility because they lead to oxidative stress (Mazzilli et al., 1995; Ko et al., 2014). Therefore, the treatment of frozen sperms with antioxidants improves sperm motility and the integrity of sperm plasma membranes, reduces the degree of oxidation of sperm plasma membranes, and protects male reproductive organs and cells of mammals thereby helping the improvement of the reproductive ability of sperms (Jang et al., 2007; Lee et al., 2017).

Vitamin E, melatonin, silymarin, and curcumin, which are used as representative antioxidants, have been reported to reduce lipid peroxidation of sperms when added to the cryopreservation solution in the cryopreservation of pig sperms (Kim and Lee et al., 2017).
Chitosan is a copolymer of $\mathrm{N}$-acetyl-glucosamine and N-glucosamine are obtained from chitin, which is widely distributed in the natural world, such as the shells of crustacean such as crabs and shrimp, the epidermis and outer shells of insects such as locusts, and the cell walls of fungi such as mushrooms and yeast. It is a natural product-derived material that can be obtained from the natural world and has the advantage of being biodegradable and nontoxic (Shin et al., 2004). The Chitosan indicates higher oral absorption with various biological effects such as anti-microbial, anti-tumor and anti-oxidant effects (Aam et al., 2010; Chung et al., 2012).

Study findings indicating that chitosan as such reduces the oxidative stress induced in sperms and testis tissues and protects cells against DNA damage caused by reactive oxygen species have been steadily reported (Chakraborty et al., 2011; Nadapdap et al., 2014; Ghanbari et al., 2018).

The present study expected that chitosan complexes added to the cryopreservation solution in the cryopreservation of sperms would have affirmative effects on sperm motility and the degree of lipid peroxidation. Accordingly, the aim of this study was to assess the post-thawed sperm kinetics and antioxidant properties of cryoprotectant supplemented with chitosan complex.

\section{MATERIALS AND METHODS}

\section{Semen collection}

The present study used pig semen collected from PWG miniature pigs raised at Kangwon National University. The semen was collected from male pigs by the manual penis pressing method using a dummy, and was transported to the laboratory while being maintained at $17^{\circ} \mathrm{C}$. Those semen samples that showed sperm motility not lower than $80 \%$ and progressive motility not lower than $70 \%$ were used after confirming the conditions using computer assisted sperm analysis (CASA, Hamilton Thorne, Inc., HTM-HELOS, Beverly, MA, USA).

\section{Chitosan preparation}

The GnHA (chitosan+hyaluronic acid, HA) and GnHG (chitosan hydrogel) were prepared as previously described (Park et al., 2011). Briefly, a chitosan was fully dissolved in $1 \%$ acetic acid to be a $1 \%$ solution $(\mathrm{w} / \mathrm{v})$ at room temperature, and the $\mathrm{pH}$ of the solution was adjusted to $\mathrm{pH} 5.0$ with $\mathrm{NaHCO}_{3}$. The concentration of chitosan solution was $1 \%$ in $1 \%$ acetic acid, and the concentration of HA solution was $0.1 \%$ in water. A chitosan hydrogel ( $\mathrm{GnHG}$ ) 
was readily treated by addition of a sodium hydroxide solution to a chitosan acetic acid solution.

\section{Diluent and cryopreservation solution}

Modified Modena B (mMB: $6 \mathrm{~g}$ glucose, $0.45 \mathrm{~g}$ EDTA, $1.38 \mathrm{~g}$ sodium citrate, $0.2 \mathrm{~g}$ sodium bicarbonate, $1 \mathrm{~g}$ Tris base, $0.5 \mathrm{~g}$ citric acid, $0.01 \mathrm{~g}$ cysteine, $0.8 \mathrm{~g}$ BSA and $0.06 \mathrm{~g}$ kanamycin sulfate; $\mathrm{pH}$ 7) was used as a basic diluent of pig semen, lactose-egg yolk (LEY extender: $80 \% \mathrm{v} / \mathrm{v}$ lactose solution $[310 \mathrm{mM}]$ and $20 \%$ $\mathrm{v} / \mathrm{v}$ egg yolk, $100 \mu \mathrm{g} / \mathrm{mL}$ kanamycin sulfate) was used as a primary cryopreservation solution, and LEY-glyserol-Orvus-ES-Paste (LEYGO: $89.5 \%$ v/v LEY; 9\% v/v glycerol; $1.5 \%$ v/v Orvus ES Pate [OEP]; $100 \mathrm{mM}$ trehalose; and $0,0.25,0.5,1 \mathrm{mg} / \mathrm{mL}$ GnHA or GnHG) was used as a secondary cryopreservation solution.

\section{Pig semen preparation and freezing}

The pig semen was diluted in mMB solution at a ratio of 1 : 3 and centrifuged $\left(400 \times \mathrm{g}, 10 \mathrm{~min}, 24^{\circ} \mathrm{C}\right)$, and the supernatant was removed. The semen was washed 3 to 5 times with $\mathrm{mMB}$ diluted solution according to the conditions of the semen and diluted in LEY so that the number of sperms after washing became 1 $\times 10 \% / \mathrm{mL}$. The sperm-LEY solution was cooled to $4^{\circ} \mathrm{C}$ by lowering the temperature by $1^{\circ} \mathrm{C}$ per 5 minutes in ice water. The cooled sperm-LEY solution was mixed with each of LEYGO solutions added with GnHA and GnHG to concentrations of $0,0.25,0.5$ and $1 \mathrm{mg} / \mathrm{mL}$, respectively, at a ratio of $1: 1$. The sperm-LEYGO mixed solution was put into $0.5 \mathrm{~mL}$ straws, pre-frozen for 5 minutes with the chill of liquid nitrogen at about $5 \mathrm{~cm}$ above a ice-box containing liquid nitrogen, and stored in liquid nitrogen.

\section{Thawing and sperm motility Test (CASA Analysis)}

The cryopreserved straws were thawed by immersing them in a constant-temperature water bath preheated to $50^{\circ} \mathrm{C}$ for 10 seconds. The thawed semen was diluted by putting it into a 15 $\mathrm{mL}$ tube containing $5 \mathrm{~mL} \mathrm{mMB}$ preheated to $37^{\circ} \mathrm{C}$, centrifuged (9,000 rpm, 3min), and the foregoing was repeated once again.
After diluting the semen with $500 \mu \mathrm{L}$ of $\mathrm{mMB}, 3 \mu \mathrm{L}$ of the semen was put into a Leja slide preheated to $37^{\circ} \mathrm{C}$ to observe sperms under a microscope. Sperm motility test was conducted with computer assisted sperm analysis (CASA) and sperm counts, motility, progressive motility, VAP (path velocity), VSL (straightline velocity), and VCL (curvilinear velocity) were observed.

\section{Measurement of lipid peroxidation of sperms}

To determine the degree of lipid peroxidation of the frozen semen, MDA values were measured using the Lipid Peroxidation (MDA) Colorimetric /Fluorometric Assay Kit (BioVision, Mountain View, CA, USA) according to the manufacturer's instructions. The thawed semen was centrifuged, the supernatant was removed, the number of sperms was adjusted to $1 \times 10^{7} / \mathrm{mL}$, and the semen was washed with PBS. The sperm pellets were dissolved in 300 $\mu \mathrm{L}$ MDA Lysis Buffer, centrifuged $(13,000 \times \mathrm{g}, 10 \mathrm{~min})$, and incubated for $60 \mathrm{~min}$ at $95^{\circ} \mathrm{C}$ to make $200 \mu \mathrm{L}$ of the supernatant react with $600 \mu \mathrm{L}$ of TBA. After the Incubation process, the tube was cooled to room temperature for $10 \mathrm{~min}$ in an ice bath and the absorbance was measured at $532 \mathrm{~nm}$. The resultant MDA value was shown to be $\mathrm{nmol} / 1 \times 10^{7}$ sperm.

\section{Statistical analysis}

The statistics of all the data used in the present study were obtained by analyses of variance through the SPSS statistics program (IBM SPSS Statistics 22), and the significant difference in the resultant data values was shown to be $p<0.05$.

\section{RESULTS}

\section{Test of the motility of pig sperms (CASA)}

The sperms frozen after being added with GnHA were thawed and analyzed and as a result, all the sperms showed a tendency to increase compared to the control as shown in Figure 1 (a), (b) and Table 1 . However, the sperms treated with $0.5 \mathrm{mg} / \mathrm{mL}$

Table 1. Effect of GnHA on sperm parameters and lipid peroxidation concentration of frozen-thawed sperm (mean \pm SD).

\begin{tabular}{ccccccc}
\hline $\begin{array}{c}\text { GnHA } \\
(\mathrm{mg} / \mathrm{mL})\end{array}$ & $\begin{array}{c}\text { Motility } \\
(\%)\end{array}$ & $\begin{array}{c}\text { Progressive motility } \\
(\%)\end{array}$ & $\begin{array}{c}\text { VAP } \\
(\mu \mathrm{m} / \mathrm{sec})\end{array}$ & $\begin{array}{c}\text { VSL } \\
(\mu \mathrm{m} / \mathrm{sec})\end{array}$ & $\begin{array}{c}\text { VCL } \\
(\mu \mathrm{m} / \mathrm{sec})\end{array}$ & $\begin{array}{c}\text { Lipid peroxidation } \\
(\mathrm{nmol} / 1 \mathrm{x} \mathrm{10} \mathrm{sperm})\end{array}$ \\
0 & $13.5 \pm 4.95$ & $9.5 \pm 3.54$ & $78.50 \pm 8.63$ & $61.15 \pm 9.26$ & $121.50 \pm 2.97$ & $0.0675 \pm 0.0308$ \\
0.25 & $29.0 \pm 1.41$ & $21.0 \pm 1.41$ & $72.55 \pm 0.64$ & $52.80 \pm 0.99$ & $124.10 \pm 0.42$ & $0.0482 \pm 0.0143$ \\
0.50 & $21.5 \pm 3.54$ & $16.0 \pm 4.24$ & $70.90 \pm 6.93$ & $53.50 \pm 1.84$ & $125.25 \pm 12.80$ & $0.1278 \pm 0.0516$ \\
1.00 & $29.5 \pm 10.61$ & $16.5 \pm 7.78$ & $54.00 \pm 4.67$ & $38.95 \pm 1.91$ & $105.60 \pm 6.36$ & $0.0450 \pm 0.0081$ \\
\hline
\end{tabular}


of GnHA were shown to have somewhat lower values compared to those treated with 0.25 or $1.0 \mathrm{mg} / \mathrm{mL}$ of GnHA. In Figure 1 (c), (d), and (e), and Table 1, VAP and VSL were shown to be lower in all experimental groups treated with GnHA compared to the control and in particular, the values were relatively much lower in the group treated with $1.0 \mathrm{mg} / \mathrm{mL}$ of GnHA. In the case of VCL, all the experimental groups had values similar to those of the control, but as with VAP and VSL, the values were shown to be relatively much lower in the group treated with $1.0 \mathrm{mg} / \mathrm{mL}$ of GnHA.

The mobility of the sperms frozen after being added with $\mathrm{GnHG}$ was checked and as a result, it can be seen that all the five resultant values were higher in the experimental group added with $0.5 \mathrm{mg} / \mathrm{mL}$ of GnHG compared to the control from Figure 2 (a) - (e) and Table 2. In the other experimental groups, all of the values generally showed a decreasing tendency but the differences were insignificant.

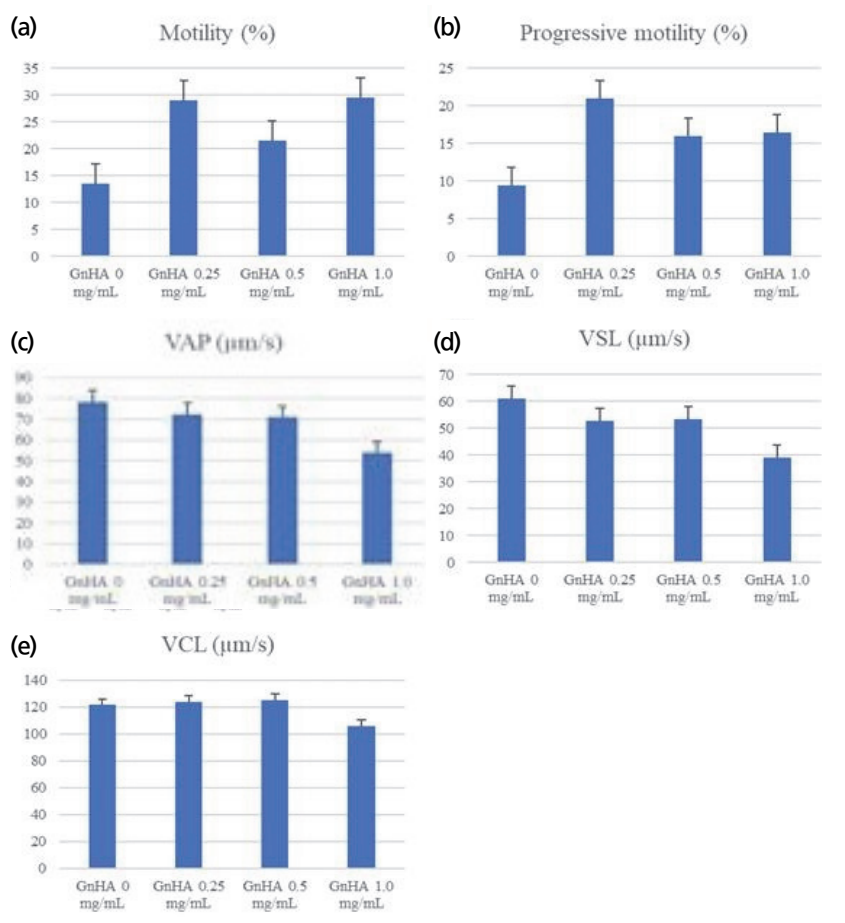

Figure 1. Sperm parameter at GnHA of cryopreservation measured by CASA.
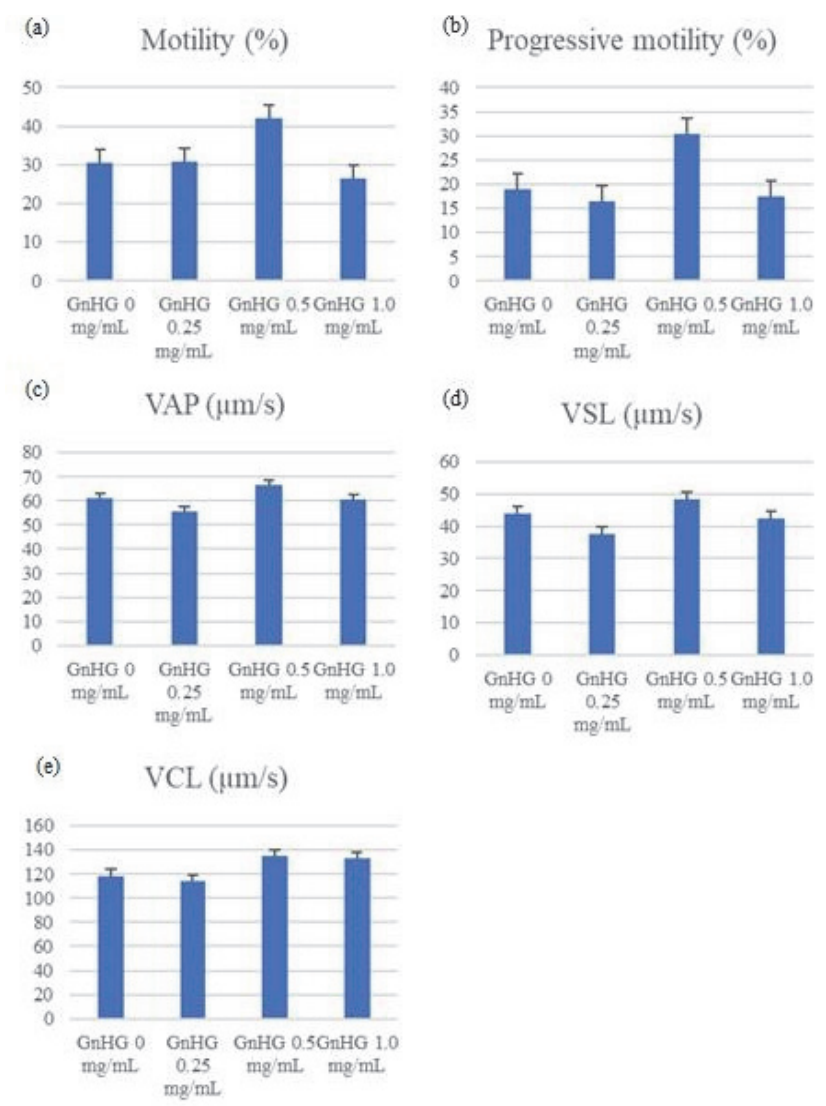

Figure 2. Sperm parameter at GnHG of cryopreservation measured by CASA.

\section{Measurement of pig sperm lipid peroxidation assay (MDA)}

Malondialdehyde assay was used to measure the degree of lipid peroxidation of pig sperms. In Figure 3 (a), it can be seen that the degree of lipid peroxidation decreased in other experimental groups than GnHA $0.5 \mathrm{mg} / \mathrm{mL}$ group compared to the control although the differences were not significant $(p<0.05)$.

As can be seen in Figure 3 (b), lipid peroxidation values significantly decreased in GnHG 0.25 and $0.5 \mathrm{mg} / \mathrm{mL}$ groups $(p<0.05)$ compared to the control.

Table 2 Effect of $\mathrm{GnHG}$ on sperm parameters and lipid peroxidation concentration of frozen-thawed sperm (mean $\pm \mathrm{SD}$ ).

\begin{tabular}{ccccccc}
\hline $\begin{array}{c}\text { GnHG } \\
(\mathrm{mg} / \mathrm{mL})\end{array}$ & $\begin{array}{c}\text { Motility } \\
(\%)\end{array}$ & $\begin{array}{c}\text { Progressive motility } \\
(\%)\end{array}$ & $\begin{array}{c}\text { VAP } \\
(\mu \mathrm{m} / \mathrm{sec})\end{array}$ & $\begin{array}{c}\text { VSL } \\
(\mu \mathrm{m} / \mathrm{sec})\end{array}$ & $\begin{array}{c}\text { VCL } \\
(\mu \mathrm{m} / \mathrm{sec})\end{array}$ & $\begin{array}{c}\text { Lipid peroxidation } \\
\left(\mathrm{nmol} / 1 \mathrm{x} 10^{7} \mathrm{sperm}\right)\end{array}$ \\
0 & $30.5 \pm 0.71$ & $19.0 \pm 1.41$ & $61.05 \pm 0.21$ & $44.1 \pm 1.84$ & $118.50 \pm 2.12$ & $0.0729 \pm 0.0063^{\mathrm{a}}$ \\
0.25 & $31.0 \pm 9.90$ & $16.5 \pm 7.78$ & $55.6 \pm 1.56$ & $37.7 \pm 2.83$ & $114.35 \pm 1.77$ & $0.0470 \pm 0.0071^{\mathrm{b}}$ \\
0.50 & $42.0 \pm 9.90$ & $30.5 \pm 12.02$ & $66.45 \pm 12.94$ & $48.5 \pm 10.89$ & $134.60 \pm 13.29$ & $0.0344 \pm 0.0053^{\mathrm{b}}$ \\
1.00 & $26.5 \pm 7.78$ & $17.5 \pm 7.78$ & $60.65 \pm 7.99$ & $42.4 \pm 5.52$ & $133.40 \pm 9.19$ & $0.3424 \pm 0.1531^{\mathrm{c}}$ \\
\hline
\end{tabular}

$\overline{\mathrm{a} \sim \mathrm{c}}$ Different superscripts within the same column indicate significant difference $(p<0.05)$. 

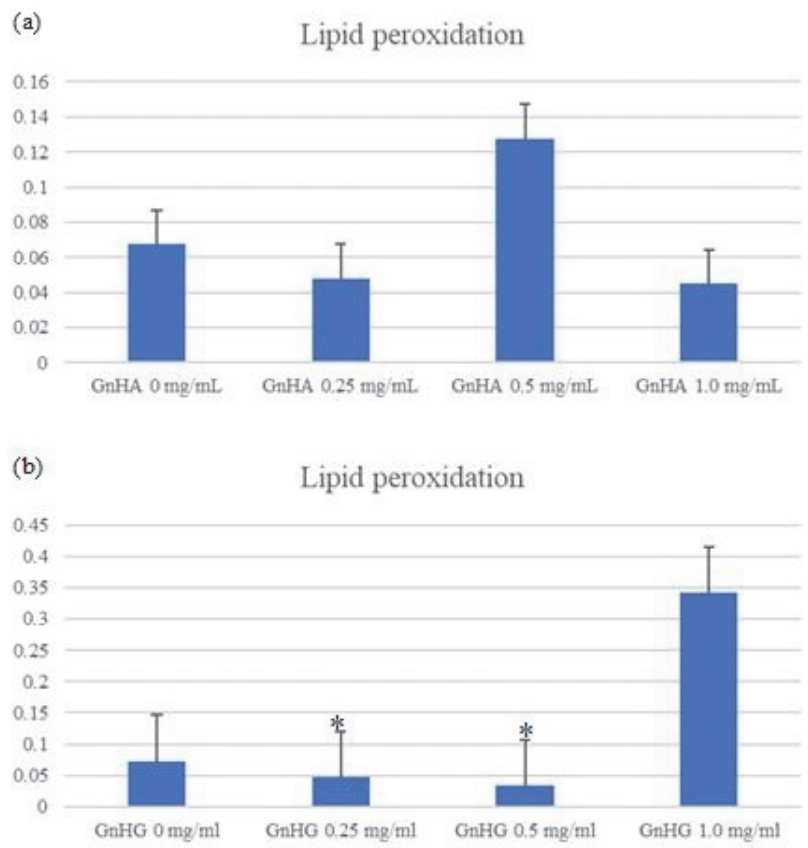

Figure 3. Lipid peroxidation assay: effect of GnHA (a) and GnHG (b) at boar sperm cryopreservation.

\section{DISCUSSION}

The present study was conducted to add chitosan complexes to the cryopreservation solution in the cryopreservation of pig sperms and thaw the sperms to check the condition of the sperms through computer-assisted sperm analysis (CASA) and lipid peroxidation assay.

CASA was used for sperm motility tests. The motility, progressive motility, path velocity (VAP), straight-line velocity (VSL), and curvilinear velocity (VCL) were observed. The motility refers to the ratio of those sperms that have the ability to move spontaneously to the entire sperms, and the progressive motility refers to the ratio of those sperms that have the ability to go straightly forward to the moving sperms. VAP means the sperm velocity in the mean path when the mean of the actual paths of sperms were obtained. VSL and VCL mean the sperm velocity in the shortest path of the distance moved by sperms and the sperm velocity in the actual path of movement, respectively.

In cases where GnHA was added as an additive in sperm cryopreservation, both the motility and progressive motility showed a tendency to increase compared to those of the control not added with GnHA. However, it can be seen that VAP, VSL, and VCL were generally similar or decreased. This suggests that GnHA acts effectively on sperm motility, but has little or no effect on the velocity.

When GnHG was added to the cryopreservation solution in the cryopreservation of sperms, it can be seen that GnHG had positive effects of both sperm motility and velocity at a concentration of $0.5 \mathrm{mg} / \mathrm{mL}$. However, it was found that quite similar values were shown when GnHG was added at other concentrations. This suggests that treatment of sperms with $0.5 \mathrm{mg} / \mathrm{mL}$ of GnHG in cryopreservation might have the effect to increase sperm activity when sperms are thawed and used.

These results suggest that the sperms cryopreserved after being added with GnHG are more effective in reducing active oxygen compared to those cryopreserved only with the cryopreservation solution.

The present study was conducted to identify the effect of sperm cryopreservation agents added with GnHG or GnHG, which is a sort of chitosan derived from natural products. CASA and lipid peroxidation assay (MDA) were used to measure the motility and lipid peroxidation of pig sperms and the results shown in Figure 1, 2, 3 and Tables 1 and 2 were obtained.

In the case of GnHA, VAP, VSL, and VCL were similar or slightly decreased compared to the control, but it can be seen that the motility and progressive motility increased in all the experimental groups added with GnHA. This seems to have similar tendencies to the results of measurement of lipid peroxidation in which lipid peroxidation decreased in the experimental groups compared to the control. Therefore, the addition of 0.25 or 1.0 $\mathrm{mg} / \mathrm{mL}$ of GnHA in sperm cryopreservation seems to enable effective use of sperms after thawing. In the case of $\mathrm{GnHG}$, the values of sperms added with of $0.5 \mathrm{mg} / \mathrm{mL}$ of $\mathrm{GnHG}$ were shown to be higher in all motility test scales. In addition, in the lipid peroxidation assay, the sperms added with of $0.5 \mathrm{mg} / \mathrm{mL}$ of $\mathrm{GnHG}$ showed significant decreases thereby showing the same tendency in both experiments. Therefore, the addition of $0.5 \mathrm{mg} / \mathrm{mL}$ of chitosan hydrogel in sperm cryopreservation is expected to have ameliorative effects on the mobility and velocity after sperm thawing so that the sperms can be more effectively used.

\section{ACKNOWLEDGEMENTS}

The authors would like to gratefully thank Prof. Jae Kweon Park, Gachon university, for providing the chitosan samples and Prof. Choon Keun Park, Kangwon national University, for semen supply. This work was supported by the National Research Foundation of Korea (NRF) and Center for Women In Science, Engineering and Technology (WISET) grant funded by the Ministry 
of Science and ICT (MSIT) under the team research program for female engineering students.

\section{REFERENCES}

Aam BB, Heggset EB, Norberg AL, Sorlie M, Varum KM and Eijsink VG. 2010. Production of chitooligosaccharides and their potential applications in medicine. Mar Drugs 8:1482-1517. Chakraborty SP, Mahapatra SK, Sahu SK, Pramanik P and Roy S. 2011. Antioxidative effect of folate-modified chitosan nanoparticles. Asian Pac. J. Trop. Biomed. 1:29 - 38.

Chung MJ, Park JK and Park YI. 2012. Anti-inflammatory effects of low-molecular weight chitosan oligosaccharides in IgE antigen complex-stimulated RBL-2H3 cells and asthma model mice. Int Immunopharmacol. 12:453-459.

Ghanbari E, Khazaei MR, Ahangar P and Khazaei M. 2018. Crab Shell Extract Improves Sperm Parameters and Antioxidant Status in Testes of Diabetic Rats. J. Diet. Suppl. 9:1-12.

Jang HY, Park CK, Cheong HT, Kim JT, Lee HK, Im SK and Yang BK. 2007. Antioxidative Effect of Pyruvate, Taurine and Melatonin, and Relationships of the Evaluation Methods on Boar Sperm Viability for In Vitro Storage. J. Anim. Sci. \& Technol. 49:729-736.

Kim HS, Lee YS, Lee SH, Cheong HT, Park CK, Lee SH and Yang BK. 2017. Effects of Arsenite and Antioxidants on Sperm Motility, Plasma Membrane Integrity, Mitochondrial Activity, and Lipid Peroxidation in Pigs. J. Life Sci. 27:517-523.

Ko EY, Sabanegh ES Jr and Agarwal A. 2014. Male infertility testing: reactive oxygen species and antioxidant capacity. Fertil. Steril. 102:1518-1527.

Lee AS, Lee SH, Lee SH and Yang BK. 2017. Effects of Curcumin on Sperm Motility, Viability, Mitochondrial Activity and Plasma Membrane Integrity in Boar Semen. Biomed. Sci. Lett. 23:406-410.

Mazzilli F, Rossi T, Sabatini L, Pulcinelli FM, Rapone S, Dondero F and Gazzaniga PP. 1995. Human sperm cryopreservation and reactive oxygen species (ROS) production. Acta Eur. Fertil. 26:145-148.

Nadapdap TP, Lutan D, Arsyad KHM and Ilyas S. 2014. Influence of Chitosan from Shrimp Skin to Quality and Quantity of Sperm of Albino Rats after Administration of Lead. Andrology $3: 114$.

Park JK, Chung MJ, Choi HN and Park YI. 2011. Effects of molecular weight and the degree of deacetylation of chitosan oligosaccharides on antitumor activity. Int $\mathrm{J}$ Mol Sci, 12:266-277

Prather RS, Hawley RJ, Carter DB, Lai L and Greenstein JL. 2003. Transgenic swine for biomedicine and agriculture. Theriogenology 59:115-123.

Safranski TJ, Ford JJ, Rohrer GA and Guthrie HD. 2011. Plenary Contribution to International Conference on Boar Semen Preservation 2011. Genetic Selection for Freezability and its Controversy with Selection for Performance. Reprod. Domest. Anim. 2:31-34.

Schäfer J, Waberski D, Jung M and Schulze M. 2017. Impact of holding and equilibration time on post-thaw quality of shipped boar semen. Anim. Reprod. Sci. 187:109-115.

Shin SY, Park HN, Kim KH, Lee SJ, Park YJ, Ku Y, Rhyu IC, Han SB and Chung CP. 2004. A study on the biodegradable novel chitosan nanofiber membrane as a possible tool for guided bone regeneration. J. Korean Acad. Periodontol. 34:543-549.

Yeste M. 2016. Sperm cryopreservation update: Cryodamage, markers, and factors affecting the sperm freezability in pigs. Theriogenology 85:47-64.

Yun IJ. 2016. Current Status of Solid Organ Xenotransplantation. J. Korean Soc. Transplant. 30:69-76. 